\title{
Au Nanoflowers for Catalyzing and In Situ Surface-Enhanced Raman Spectroscopy Monitoring of the Dimerization of $p$-Aminothiophenol
}

\author{
Jingwen Ba, Yandong Han, Xiaoyu Zhang, Lijuan Zhang, Shuhan Hui, Zhenzhen Huang,* \\ and Wensheng Yang*
}

Cite This: ACS Omega 2021, 6, 25720-25728

Read Online

ABSTRACT: In this work, we demonstrated a facile approach for fabrication of $\mathrm{Au}$ nanoflowers (Au NFs) using an amino-containing organosilane, 3-aminopropyltriethoxysilane (APTES), as a shape-directing agent. In this approach, the morphology of the Au particles evolved from sphere-like to flower-like with increasing the concentration of APTES, accompanied by a red shift in the localized surface plasmon resonance peak from 520 to $685 \mathrm{~nm}$. It was identified that the addition of APTES is profitable to direct the preferential growth of the (111) plane of face-centered cubic gold and promote the formation of anisotropic Au NFs. The as-prepared Au NFs, with APTES on their surface, presented effective catalytic and surface-enhanced Raman scattering (SERS) performances, as evidenced by their applications in catalyzing the dimerization of $p$-aminothiophenol and monitoring the reaction process via in situ SERS analysis.

\section{INTRODUCTION}

$\mathrm{Au}$ nanoflowers (Au NFs) have aroused particular interests owing to the existence of "hot spots" distributed on their rough surface and thus a significantly enhanced electromagnetic field around the junctions and sharp tips, beneficial for their applications in catalysis and surface-enhanced Raman scattering (SERS). ${ }^{1-12}$ Usually, surfactants are necessary to be employed as shape-directing agents to direct the preferential growth of the Au particles, along a definite lattice plane of facecentered cubic (fcc) gold, to form the anisotropic $\mathrm{Au}$ NFs. ${ }^{13-20}$ For example, Wang et al. reported the growth of anisotropic Au NFs by passivating the (111) facet of fcc gold using (1-hexadecyl)trimethylammonium chloride as a "faceblocking" agent. ${ }^{21}$ Hwang et al. reported the fabrication of $\mathrm{Au}$ NFs with broad NIR adsorption by directing the preferential growth along the (111) plane of fcc gold using a gemini cationic surfactant, $N, N, N^{\prime} N^{\prime}$-tetramethyl- $N, N^{\prime}$-ditetradecylethane-1,2-diaminium bromide. ${ }^{22}$ Sadik et al. demonstrated the synthesis of $\mathrm{Au}$ NFs with tunable surface roughness using pyromellitic dianhydride- $p$-phenylene diamine as both shapedirecting and reducing agents. ${ }^{23}$ Kim et al. demonstrated a covalently capped seed-mediated growth approach for the synthesis of flower-like $\mathrm{Au}$ nanostructures by employing 6mercaptohexanol and cetyltrimethylammonium bromide as shape-directing agents. ${ }^{24}$

3-Aminopropyltriethoxysilane (APTES) is an amino-containing organosilane, which is commonly used for the modification of the silica surface and can provide active amino sites for anchoring or growth of Au particles. ${ }^{25-27}$ In this work, we demonstrated a facile approach for the synthesis of $\mathrm{Au}$ NFs using APTES as a shape-directing agent. In this approach, the morphology of the Au particles evolved from sphere-like to flower-like with increasing the concentration of APTES introduced into the reaction solutions, accompanied with a red shift of the localized surface plasmon resonance (LSPR) peak $\left(\lambda_{\max }\right)$ from 520 to $685 \mathrm{~nm}$, attributed to the promoted preferential growth of the (111) plane of fcc gold by APTES. The resulting Au NFs presented effective catalytic and SERS performances, ${ }^{28}$ making them act as both the catalyst to promote the dimerization of $p$-aminothiophenol and as an in situ SERS substrate to monitor the reaction process.

\section{RESULTS AND DISCUSSION}

In this work, an amino-containing organosilane agent, APTES, was used to direct the growth of Au NFs. In this approach, first, APTES was added in water to undergo self-catalyzed hydrolysis and condensation ${ }^{29,30}$ to form oligomers, such as dimers and trimers. Then, $\mathrm{HAuCl}_{4}$ was added in the APTES solution, followed by the addition of a reductant (AA) to form the Au NFs (Scheme 1). In our experiments, first conductivity measurements were carried out to evaluate the hydrolysis and condensation of APTES in an aqueous solution. ${ }^{31}$ An aqueous solution of APTES $(3 \mathrm{mM})$ presented a conductivity of $82 \mu \mathrm{S}$. $\mathrm{cm}^{-1}$ after $10 \mathrm{~s}$ of the reaction, which dropped sharply to 48 $\mu \mathrm{S} \cdot \mathrm{cm}^{-1}$ and then remained less changed after $3 \mathrm{~min}$ of the

Received: July 23, 2021

Accepted: September 8, 2021

Published: September 21, 2021 
Scheme 1. Schematic Illustration of the Synthesis Process of the Au NFs
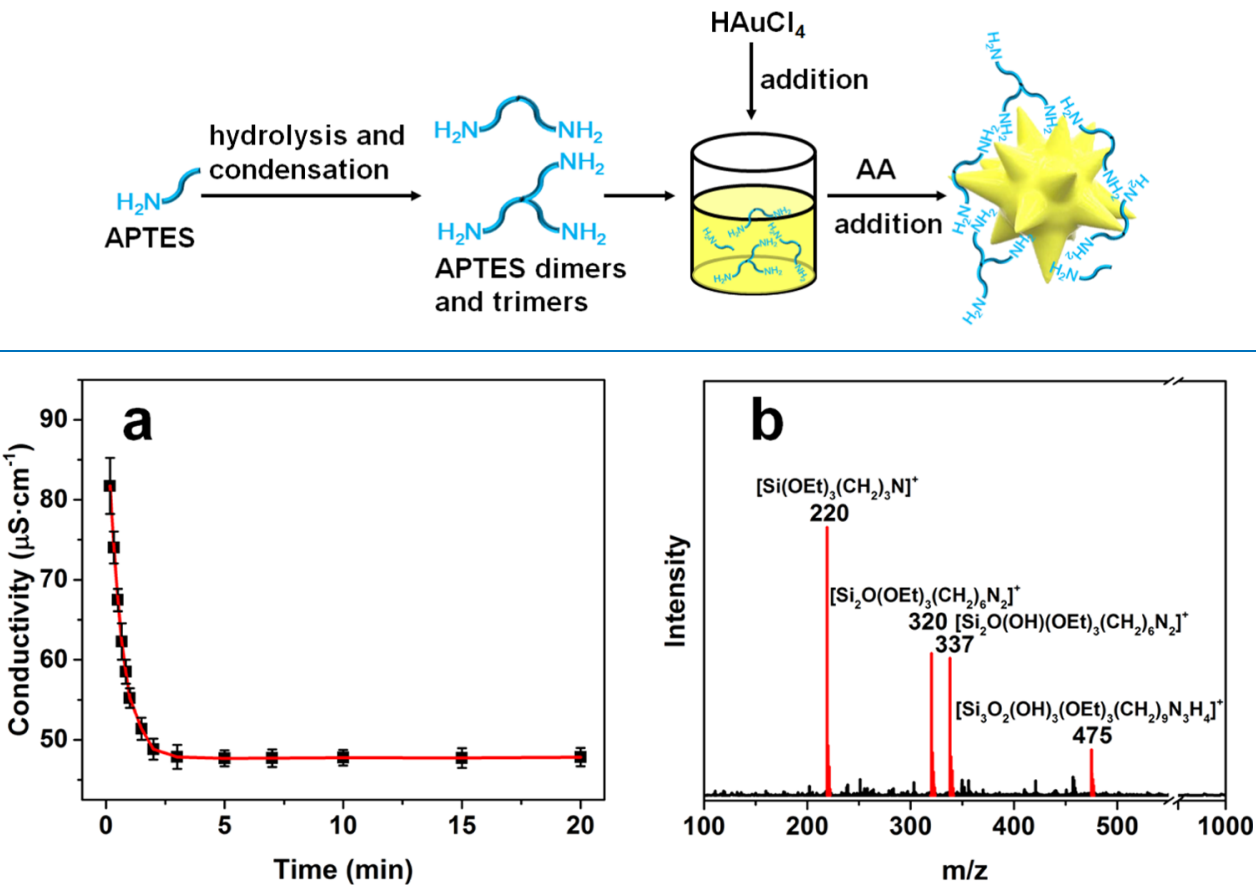

Figure 1. (a) Temporal evolution in the conductivity of the aqueous solution of APTES ( $3 \mathrm{mM}$ ). (b) Mass spectrum of the $3 \mathrm{mM}$ APTES solution taken out at $5 \mathrm{~min}$.

reaction (Figure 1a), suggesting the much fast hydrolysis over the condensation of APTES at the initial stage of the reaction $(0-3 \mathrm{~min})$ and the well-balanced hydrolysis and condensation at the late stage of the reaction $(3-20 \mathrm{~min}) .^{32}$ According to the negative differentiation curve of the conductivity (Figure $\mathrm{S} 1$ ), the maximum condensation rate of APTES was derived to be $\geq 46 \mu \mathrm{S} \cdot \mathrm{cm}^{-1} \cdot \mathrm{min}^{-1}$. ${ }^{33}$ The mass spectrum of the solution, taken out after 5 min upon the addition of APTES, presented a peak corresponding to unhydrolyzed APTES, [Si$\left.(\mathrm{OEt})_{3}\left(\mathrm{CH}_{2}\right)_{3} \mathrm{~N}\right]^{+}(\mathrm{m} / z=220)$, as well as peaks corresponding to a dimeric product with one silanol group, $\left[\mathrm{Si}_{2} \mathrm{O}(\mathrm{OH})\right.$ $\left.(\mathrm{OEt})_{3}\left(\mathrm{CH}_{2}\right)_{6} \mathrm{~N}_{2}\right]^{+}(\mathrm{m} / z=337)$ or without a silanol group, $\left[\mathrm{Si}_{2} \mathrm{O}(\mathrm{OEt})_{3}\left(\mathrm{CH}_{2}\right)_{6} \mathrm{~N}_{2}\right]^{+}(\mathrm{m} / z=320)$. In addition, a peak corresponding to a trimeric product with three silanol groups, $\left[\mathrm{Si}_{3} \mathrm{O}_{2}(\mathrm{OH})_{3}(\mathrm{OEt})_{3}\left(\mathrm{CH}_{2}\right)_{9} \mathrm{~N}_{3} \mathrm{H}_{4}\right]^{+}(\mathrm{m} / z=475)$, was also observed in the spectrum (Figure $1 \mathrm{~b}$ ). These results indicated that APTES undergoes hydrolysis and the hydrolyzed APTES condenses into dimers and trimers in the aqueous solution, catalyzed by its own amino group. ${ }^{34}$ Therefore, in our experiments, the aqueous solution of APTES was kept at room temperature for $5 \mathrm{~min}$, allowing the hydrolysis and condensation of APTES to reach the balance, before mixing it with the aqueous solution of $\mathrm{HAuCl}_{4}$.

In the subsequent procedure, the aqueous solution of $\mathrm{HAuCl}_{4}(100 \mathrm{mM})$ was mixed with different volumes of the APTES solution ( $3 \mathrm{mM})$, prehydrolyzed at room temperature for $5 \mathrm{~min}$, and then $\mathrm{AA}$ was added into the mixture to initiate the reduction of $\mathrm{HAuCl}_{4}$ and the growth of $\mathrm{Au}$ particles. The concentrations of $\mathrm{HAuCl}_{4}$ and $\mathrm{AA}$ were fixed at 0.25 and 1.5 $\mathrm{mM}$ unless stated especially, and that of APTES was changed in a range of $0-2.5 \mathrm{mM}$, corresponding to the molar ratio of $\mathrm{HAuCl}_{4}$ and APTES ( $\left.R_{\mathrm{Au} / \mathrm{APTES}}\right)$, in the range from 1:0 to 1:10. Figure 2 shows UV-vis spectra of the Au particles prepared with different $R_{\mathrm{Au} / \mathrm{APTES}}$ values. In the absence of APTES $\left(R_{\mathrm{Au} / \mathrm{APTES}}\right.$ of $\left.1: 0\right)$, the resulting Au hydrosol was red in color,



Figure 2. Normalized UV-vis spectra of the Au hydrosols prepared at different molar ratios of $\mathrm{HAuCl}_{4}$ and APTES ( $\left.R_{\text {Au/APTES }}\right)$ from 1:0 to $1: 10$. The inset shows photographs of the corresponding hydrosols.

corresponding to an LSPR peak with $\lambda_{\max }$ centered at $520 \mathrm{~nm}$, suggesting the formation of sphere-like Au particles. When the value of $R_{\mathrm{Au} / \mathrm{APTES}}$ was elevated to $1: 2,1: 4,1: 6,1: 8$, and $1: 10$, the resulting hydrosols became purple, light blue, blue, bluish green, and bluish gray in color, and correspondingly, $\lambda_{\max }$ of the LSPR peak shifted to $529,653,636,677$, and $685 \mathrm{~nm}$, respectively, suggesting the increased size and/or more anisotropic character of the resulting Au particles. Transmission electron microscopy (TEM) observations and the histogram of the numerical size distributions showed that the $\mathrm{Au}$ particles grown at $R_{\mathrm{Au} / \mathrm{APTES}}$ of 1:0 were sphere-like in shape, with an average size of $10 \pm 1.4 \mathrm{~nm}$ (Figure $3 \mathrm{a}$ ). When the $R_{\mathrm{Au} / \mathrm{APTES}}$ was $1: 2$, the resulting Au particles became less regular in shape, with an average size of $14 \pm 1.9 \mathrm{~nm}$ (Figure $3 \mathrm{~b}$ ). Such a slight increase in the size of the Au particles (from $10 \pm 1.4$ to $14 \pm 1.9 \mathrm{~nm}$ ) indicated that the red shift in $\lambda_{\max }$ of the LSPR peak (from 520 to $529 \mathrm{~nm}$ ) and the change in color (from red to purple) of the $\mathrm{Au}$ hydrosols are primarily 

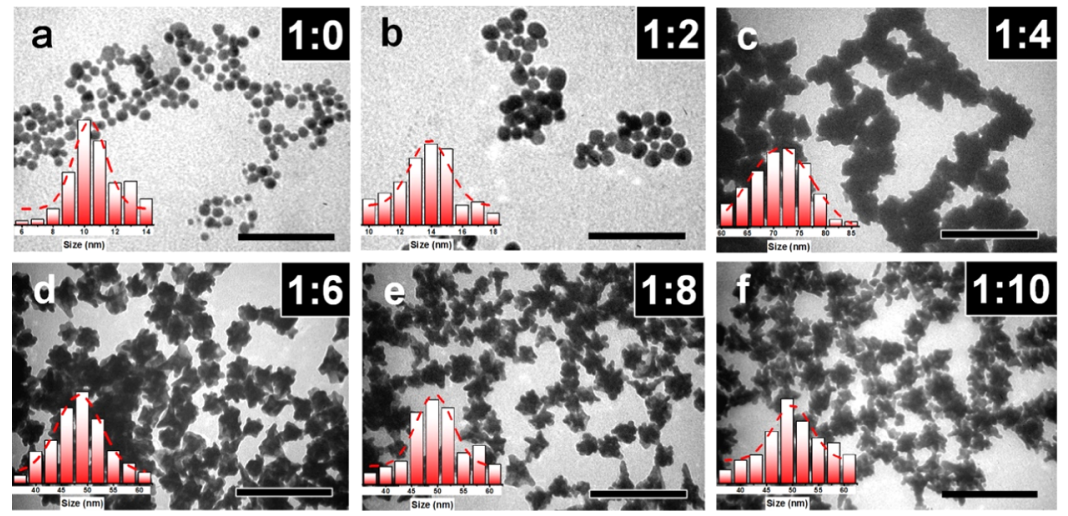

Figure 3. TEM images of the Au particles prepared at $R_{\text {Au/APTES }}$ of (a) 1:0, (b) 1:2, (c) 1:4, (d) 1:6, (e) 1:8, and (f) 1:10. The inset gives histograms of the numerical size distribution of the particles. The scale bars in TEM images are $100 \mathrm{~nm}$ in (a, b) and $200 \mathrm{~nm}$ in $(\mathrm{c}-\mathrm{f})$.
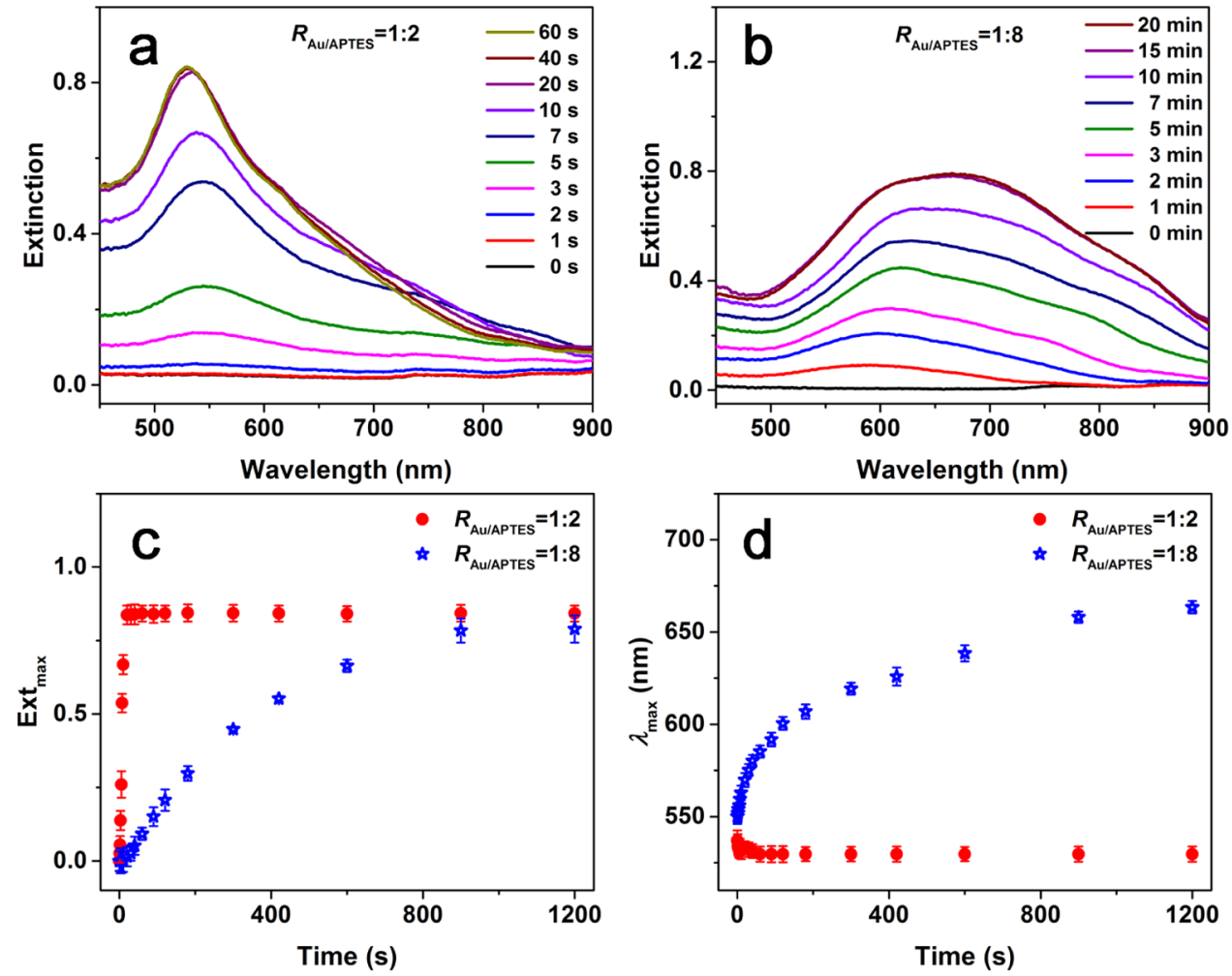

Figure 4. Temporal evolution in UV-vis spectra of the reactions carried out at $R_{\mathrm{Au} / \mathrm{APTES}}$ of (a) 1:2 and (b) 1:8. Summarized temporal evolution in (c) maximum extinction and (d) $\lambda_{\max }$ of the LSPR peak of the reaction solutions carried out at $R_{\mathrm{Au} / \mathrm{APTES}}$ of 1:2 (red) and 1:8 (blue).

attributed to the change in the morphology of the Au particles. When the value of $R_{\text {Au/APTES }}$ was elevated to $1: 4$, the resulting $\mathrm{Au}$ particles became flower-like in shape, with an average size of $73 \pm 4.1 \mathrm{~nm}$ (Figure $3 \mathrm{c}$ ). Further increasing the $R_{\mathrm{Au} / \mathrm{APTES}}$ to $1: 6,1: 8$, and $1: 10$ resulted in the formation of the Au NFs with longer branches with almost the same average size of $49 \pm 2.3$ $\mathrm{nm}$ (Figure $3 \mathrm{~d}-\mathrm{f}$ ). It was noted that the Au particles prepared at an $R_{\mathrm{Au} / \mathrm{APTES}}$ value of 1:4 presented a much red-shifted $\lambda_{\max }$ of the LSPR peak than those prepared at an $R_{\mathrm{Au} / \mathrm{APTES}}$ value of 1:6 (653 vs $636 \mathrm{~nm})$. It was likely that such a red shift in $\lambda_{\max }$ of the LSPR peak is related to the difference in the size of the $\mathrm{Au}$ NFs $(73 \pm 4.1$ vs $49 \pm 2.3 \mathrm{~nm})$ since there was no superiority in the branch length for the Au NFs prepared at an $R_{\text {Au/APTES }}$ value of $1: 4$ compared with that of the $\mathrm{Au} N F s$ prepared at an $R_{\mathrm{Au} / \mathrm{APTES}}$ value of 1:6. Based on these results, it was deduced that the increase in the $R_{\mathrm{Au} / \mathrm{APTES}}$ value facilitates the formation of $\mathrm{Au}$ NFs with an increased branch length and thus red-shifted $\lambda_{\max }$ of the LSPR peak. A control experiment was carried out to further elucidate the effect of APTES on growth of the Au NFs. When fresh APTES was used instead of the prehydrolyzed APTES in the reaction, the branch length of the $\mathrm{Au}$ NFs, formed at $R_{\mathrm{Au} / \mathrm{APTES}} 1: 8$, became shorter, accompanied by a blue shift in $\lambda_{\max }$ of the LSPR peak (677 vs $612 \mathrm{~nm}$, Figure S2), indicating that the prehydrolyzed APTES is more effective in promoting the formation of Au NFs than the fresh one.

Temporal evolutions in UV-vis absorption spectra of the reactions carried out at two typical $R_{\mathrm{Au} / \mathrm{APTES}}$ values, i.e., 1:2 and $1: 8$, were followed to further understand the effect of APTES on the morphology of the resulting Au particles. In the reaction carried out at $R_{\mathrm{Au} / \mathrm{APTES}}$ of $1: 2$, an LSPR peak with a $\lambda_{\max }$ at $535 \mathrm{~nm}$ became observable after $3 \mathrm{~s}$ of the reaction, 

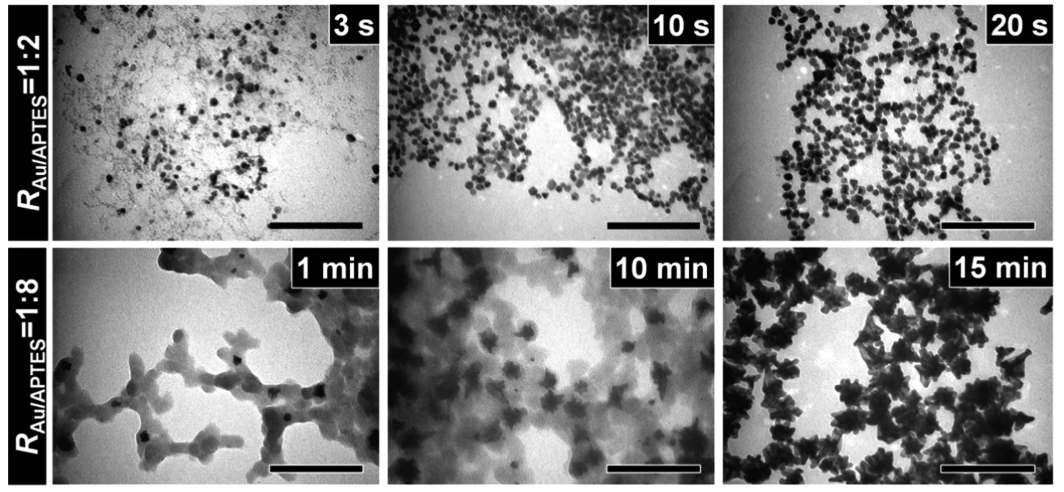

Figure 5. Temporal evolution in size/shape of the Au particles grown in the reactions carried out at $R_{\mathrm{Au} / \mathrm{APTES}}$ of (top panel) $1: 2$ and (lower panel) $1: 8$. The scale bars in all of the TEM images are $200 \mathrm{~nm}$.
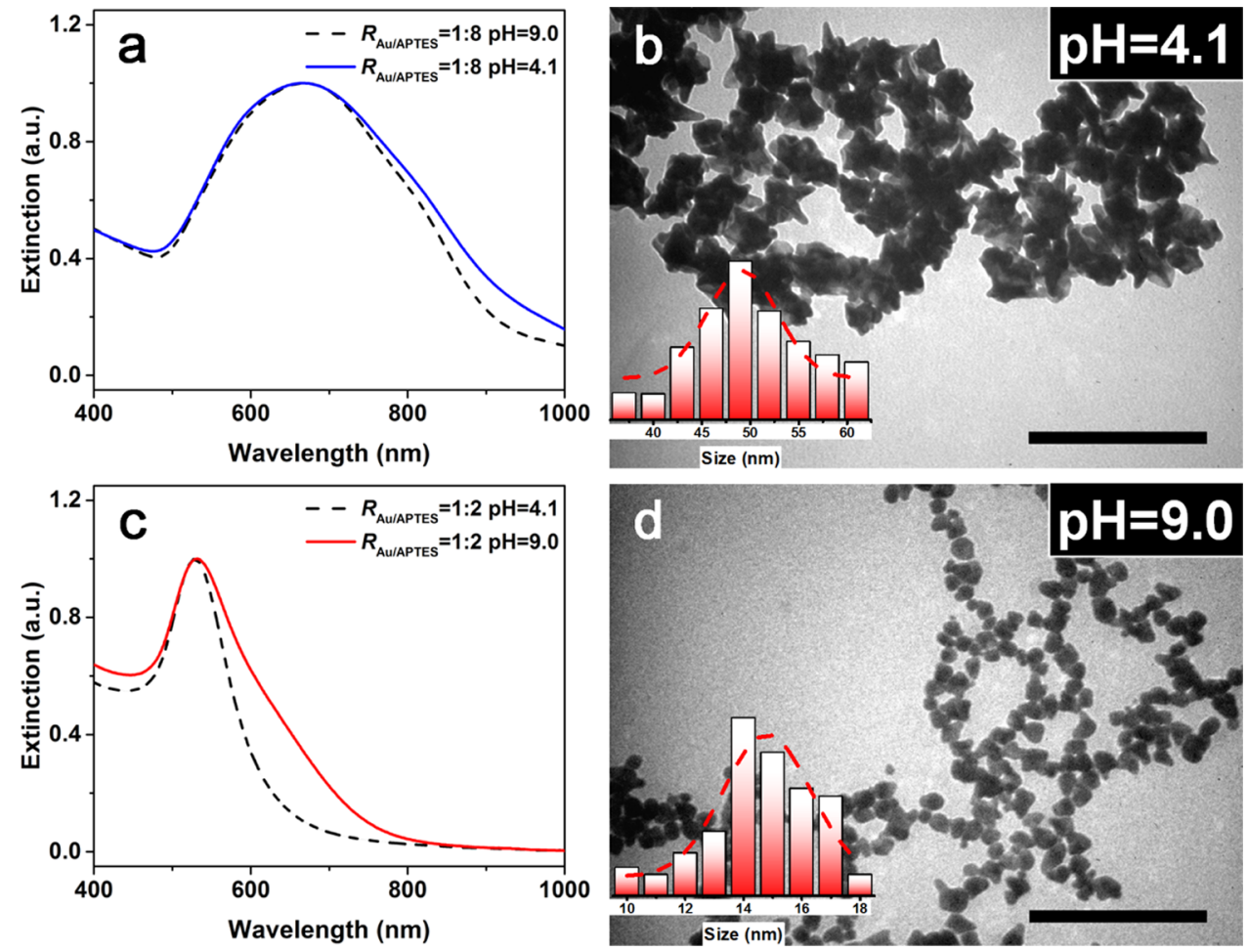

Figure 6. (a) Normalized UV-vis spectrum and (b) TEM image of the Au particles grown in the reaction carried out at $R_{\mathrm{Au} / \mathrm{APTES}}$ of $1: 8$ and $\mathrm{pH}$ 4.1. Normalized UV-vis spectrum of the Au NFs grown at the same $R_{\mathrm{Au} / \mathrm{APTES}}$ value and $\mathrm{pH} 9.0$ was given as a dotted line for comparison. (c) Normalized UV-vis spectrum and (d) TEM image of the Au particles grown in the reaction carried out at $R_{\text {Au/APTES }}$ of 1:2 and pH 9.0. Normalized $\mathrm{UV}$-vis spectrum of the Au particles grown at the same $R_{\mathrm{Au} / \mathrm{APTES}}$ value and $\mathrm{pH} 4.1$ was given as a dotted line for comparison. Insets show histograms of the numerical size distribution of the particles. The scale bars in all of the TEM images are $200 \mathrm{~nm}$.

whose intensity increased rapidly and remained almost unchanged after $20 \mathrm{~s}$ of the reaction (Figure 4a,c), accompanied by a slight blue shift in $\lambda_{\max }$ of the LSPR peak from 535 to $529 \mathrm{~nm}$ (Figure 4d). For the reaction carried out at $R_{\mathrm{Au} / \mathrm{APTES}}$ of $1: 8$, an LSPR peak with $\lambda_{\max }$ at $580 \mathrm{~nm}$ became observable after $1 \mathrm{~min}$ of the reaction, whose intensity increased gradually and then remained almost unchanged after $15 \mathrm{~min}$ of the reaction (Figure $4 \mathrm{~b}, \mathrm{c}$ ), accompanied by an obvious red shift in $\lambda_{\max }$ of the LSPR peak from 580 to $677 \mathrm{~nm}$ (Figure 4d), indicating the reduced reactivity of the gold precursor and the facilitated growth of the Au NFs under the elevated APTES concentration. TEM observations showed that in the reaction with $R_{\mathrm{Au} / \mathrm{APTES}}$ of $1: 2$, Au particles with an average size of ca. $10 \mathrm{~nm}$ were observable after $3 \mathrm{~s}$ of the reaction. When the reaction was prolonged to 10 and $20 \mathrm{~s}$, the average size of the Au particles only increased slightly to ca. 13 and $14 \mathrm{~nm}$ (Figure 5, top panel). Taking the UV-vis spectral results and the TEM observations together, it was deduced that there is continuous nucleation in the reaction carried out at this low $R_{\mathrm{Au} / \mathrm{APTES}}$ value $(1: 2)$, attributed to high activity of the gold precursor, contributing to the formation of small spherelike $\mathrm{Au}$ particles (Figure S3a). In the reaction solution with $R_{\text {Au/APTES }}$ of $1: 8$, the Au particles formed after $1 \mathrm{~min}$ of the reaction were irregular in shape, which evolved into a more flower-like morphology after $15 \mathrm{~min}$ of the reaction (Figure 5, lower panel, and Figure S3b). These results suggested the promoted formation of Au NFs at a high $R_{\mathrm{Au} / \mathrm{APTES}}$ value (1:8), possibly due to a decrease in the activity of the gold precursor and thus slowed down the growth of the Au particles at a high APTES concentration. ${ }^{7}$ 

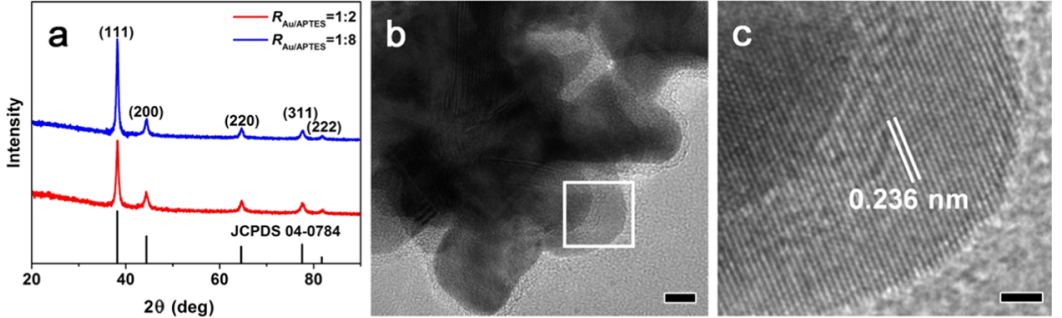

Figure 7. (a) XRD patterns of the Au particles prepared at $R_{\text {Au/APTES }}$ of $1: 2$ and 1:8. The standard XRD pattern of fcc gold (JCPDS 04-0784) is also given for comparison. (b) Enlarged TEM image of the Au NFs prepared at $R_{\mathrm{Au} / \mathrm{APTES}}$ of 1:8 and (c) HRTEM image of the labeled branch in (b). The scale bars in $(b, c)$ are 5 and $2 \mathrm{~nm}$, respectively.
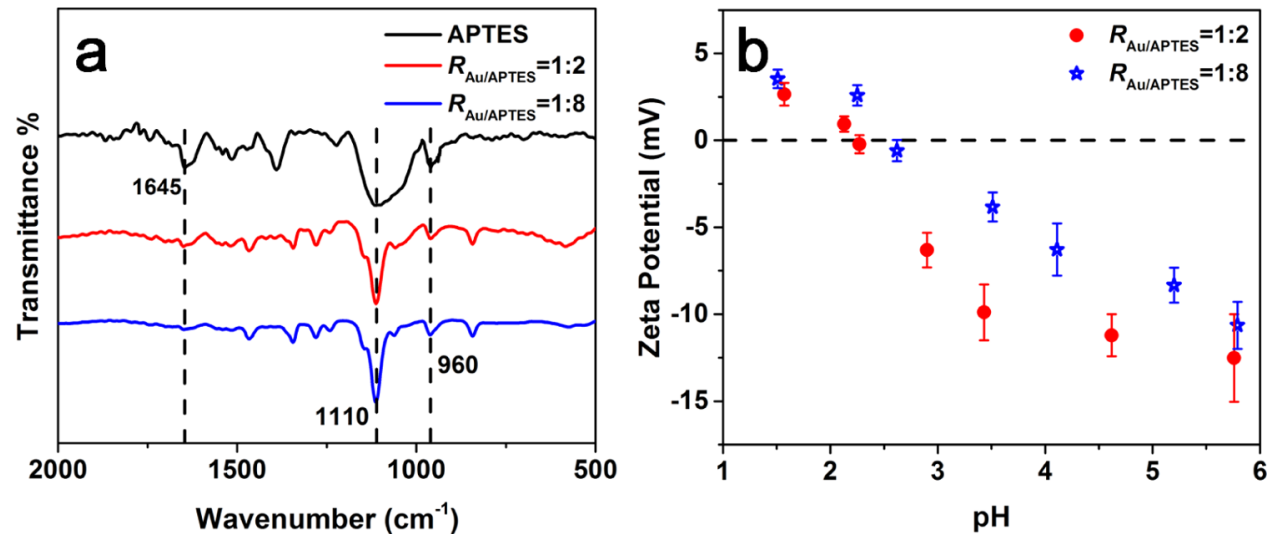

Figure 8. (a) FTIR spectra of APTES and the Au particles prepared at $R_{\mathrm{Au} / \mathrm{APTES}}$ of 1:2 and 1:8. (b) Variations in $\zeta$-potential of the Au particles prepared at $R_{\mathrm{Au} / \mathrm{APTES}}$ of $1: 2$ and 1:8 with $\mathrm{pH}$.

It was expected that the change in $R_{\text {Au/APTES }}$ may affect $\mathrm{pH}$ of the reaction solution and thus reactivity of the Au precursor since APTES is a weak base in character. When the value of $R_{\mathrm{Au} / \mathrm{APTES}}$ was set to be $1: 2$, initial $\mathrm{pH}$ of the reaction solution was measured to be 4.1 , which increased to 9.0 when the value of $R_{\mathrm{Au} / \mathrm{APTES}}$ was elevated to $1: 8$. To elucidate the effect of $\mathrm{pH}$ on growth of the $\mathrm{Au}$ particles, initial $\mathrm{pH}$ of the reaction solution with $R_{\mathrm{Au} / \mathrm{APTES}}$ of 1:8 was adjusted from 9.0 to 4.1 by addition of $\mathrm{HNO}_{3}$, and that of the reaction solution with $R_{\text {Au/APTES }}$ of $1: 8$ was adjusted from 4.1 to 9.0 by addition of $\mathrm{LiOH}$. For the reactions carried out at $R_{\mathrm{Au} / \mathrm{APTES}}$ of $1: 8, \lambda_{\max }$ of the LSPR peak remained almost unchanged (Figure $6 \mathrm{a}$ ) and the resulting $\mathrm{Au}$ particles were still flower-like in shape (Figure 6b) after $\mathrm{pH}$ of the reaction solution was lowered to 4.1. For the reactions carried out at $R_{\mathrm{Au} / \mathrm{APTES}}$ of $1: 2, \lambda_{\max }$ of the LSPR peak remained almost unchanged (Figure $6 c$ ) and the resulting $\mathrm{Au}$ particles were still primarily sphere-like in shape (Figure $6 \mathrm{~d}$ ) after $\mathrm{pH}$ of the reaction solution was elevated to 9.0. It was noted that extinction in the $600-800 \mathrm{~nm}$ window was almost unchanged when $\mathrm{pH}$ of the reaction solution with $R_{\mathrm{Au} / \mathrm{APTES}}$ of $1: 8$ was lowered from 9.0 to 4.1 , and that increased to some extent when $\mathrm{pH}$ of the reaction solution with $R_{\mathrm{Au} / \mathrm{APTES}}$ of $1: 2$ was elevated from 4.1 to 9.0 (Figure $6 \mathrm{a}, \mathrm{c}$ ), indicating the slightly promoted anisotropic character of the resulting $\mathrm{Au}$ particles, possibly due to the reduced activity of the gold precursor at elevated $\mathrm{pH}^{35,36}$ These results implied that the ratio of $R_{\mathrm{Au} / \mathrm{APTES}}$ is more effective than $\mathrm{pH}$ in tuning shape of the resulting $\mathrm{Au}$ particles since the change in $\mathrm{pH}$ was not enough to induce the change in the shape of the resulting $\mathrm{Au}$ particles at either a low $(1: 2)$ or high $(1: 8) R_{\text {Au/APTES }}$ ratio.

X-ray diffraction (XRD) patterns (Figure $7 \mathrm{a}$ ) were collected to further understand the effect of APTES on growth of the Au particles. Both the Au particles prepared at $R_{\mathrm{Au} / \mathrm{APTES}}$ of $1: 2$ and $1: 8$ presented diffraction peaks at $38.2,44.4,64.8,77.9$, and $81.7^{\circ}$, corresponding to the (111), (200), (220), (311), and (222) planes, well matched with those of the fcc gold. It was noted that the intensity ratio of the (111) and (200) planes $\left(I_{111} / I_{200}\right)$ was 3.44 for the Au particles prepared at $R_{\mathrm{Au} / \mathrm{APTES}}$ of $1: 2$, which increased to 4.35 for those prepared at $R_{\mathrm{Au} / \mathrm{APTES}}$ of $1: 8$, indicating the preferential growth of the Au particles along the (111) facet under the elevated APTES concentration, contributing to formation of the anisotropic Au NFs. ${ }^{17}$ High-resolution transmission electron microscopy (HRTEM) observations revealed that branches of the Au NFs were mainly composed of a lattice with a fringe spacing of $0.236 \mathrm{~nm}$ (Figure $7 \mathrm{~b}, \mathrm{c})$, corresponding to the (111) plane of fcc gold, consistent with the XRD results. Thus, it was concluded that the increased concentration of APTES is favorable for preferential growth of $\mathrm{Au}$ particles along the (111) plane of fcc gold, resulting in the formation of $\mathrm{Au}$ NFs.

The presence of APTES on the surface of the Au particles was revealed by Fourier transform infrared (FTIR) spectra, $\zeta$ potential, and energy dispersive X-ray spectroscopy (EDS) measurements. The stretching vibrations of $\mathrm{Si}-\mathrm{OH}$ at 960 $\mathrm{cm}^{-1}$ and $\mathrm{Si}-\mathrm{O}-\mathrm{Si}$ at $1110 \mathrm{~cm}^{-1,30,37}$ attributed to the condensed and/or hydrolyzed APTES, were identifiable in the $\mathrm{Au}$ particles prepared at either a low (1:2) or high (1:8) $R_{\text {Au/APTES }}$ ratio, suggesting the existence of APTES on the particle surfaces. It was noted that the $\mathrm{N}-\mathrm{H}$ bending vibration of APTES at $1645 \mathrm{~cm}^{-1}$ became weaker in the presence of the $\mathrm{Au}$ particles (Figure 8a), indicating that APTES is anchored onto the particle surface via its amino group. ${ }^{38}$ The flower-like Au particles, prepared at an $R_{\mathrm{Au} / \mathrm{APTES}}$ ratio of $1: 8$, presented an isoelectric point around 2.8, similar to that (ca. 2.5) of the 

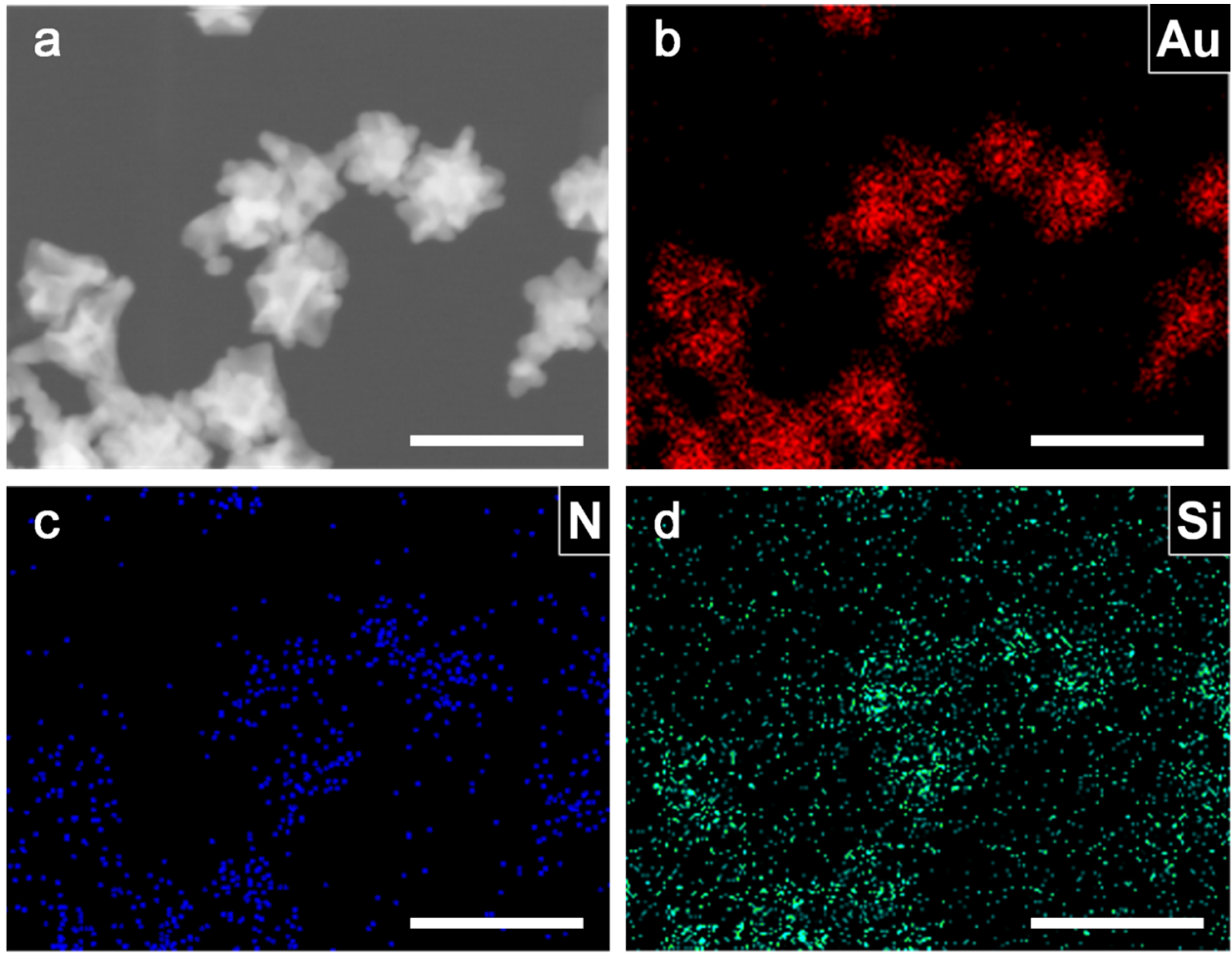

Figure 9. (a) STEM image of the Au NFs prepared at $R_{\text {Au/APTES }}$ at 1:8 and the corresponding element mapping images of (b) Au, (c) N, and (d) Si. The scale bars in all of the images are $100 \mathrm{~nm}$.
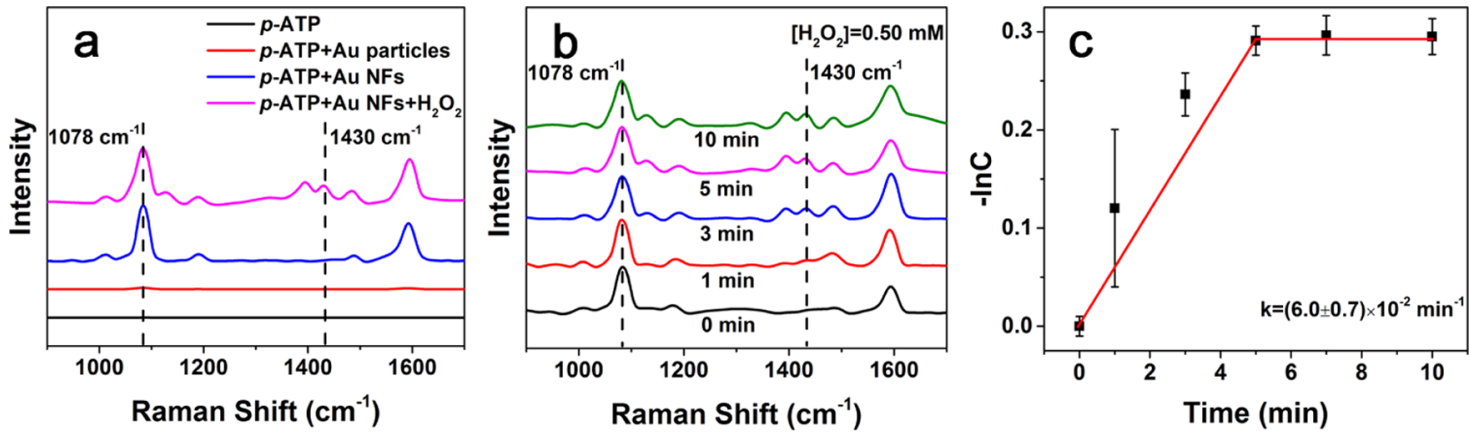

Figure 10. (a) SERS spectra of $1.0 \mu \mathrm{M} p$-ATP in the presence of the Au particles prepared at $R_{\text {Au/APTES }}$ of $1: 2$ (red) and the Au NFs prepared at $R_{\text {Au/APTES }}$ of 1:8 in the absence (blue) and the presence (purple) of $\mathrm{H}_{2} \mathrm{O}_{2}$. The Raman spectrum of the $0.2 \mathrm{mM} p$-ATP solution (black) is also given for comparison. (b) Normalized time-dependent SERS spectra of $1.0 \mu \mathrm{M} p$-ATP in the presence of the Au NFs prepared at $R_{\text {Au/APTES }}$ of 1:8 and $0.50 \mathrm{mM} \mathrm{H}_{2} \mathrm{O}_{2}$. (c) Corresponding fitting curve of the negative natural logarithm of the estimated relative concentration of $p$-ATP vs the reaction time derived from $(\mathrm{b})$.

sphere-like Au particles prepared at the $R_{\text {Au/APTES }}$ ratio of $1: 2$ (Figure $8 \mathrm{~b}$ ), indicating the similar surface character of the flower-like and sphere-like Au particles. ${ }^{39}$ Scanning transmission electron microscopy (STEM) and the corresponding EDS elemental mapping images revealed the coexistence of $\mathrm{Au}$, $\mathrm{N}$, and Si elements on the surface of the Au NFs (Figure 9), further proving the existence of APTES on the Au particle surface.

The stability of the Au NFs was evaluated by dynamic light scattering and UV-vis spectra. After being kept at room temperature for $60 \mathrm{~h}$, there were almost no changes in hydrodynamic diameters and polydispersity, as well as UV-vis spectra, of the Au NFs prepared at $R_{\mathrm{Au} / \mathrm{APTES}}$ of 1:8 (Figure S4), indicating excellent stability of the Au NFs, attributed to the protection effect of the amino-containing organosilane (APTES) toward the Au NFs. To evaluate the reproducibility of our method, five batches of the Au NFs were prepared at $R_{\mathrm{Au} / \mathrm{APTES}}$ of 1:8. The Au NFs, prepared from the five different batches, presented very similar hydrodynamic diameters and an LSPR peak with almost the same $\lambda_{\max }$ (Figure S5), indicating the good reproducibility of the APTES-directed approach for the synthesis of Au NFs.

Dimerization of $p$-aminothiophenol ( $p$-ATP) into dimercaptoazobenzene (DMAB), a reaction with great significance in the fields of pharmaceuticals, dyes, food additives, etc., ${ }^{40}$ was employed as a model reaction to evaluate catalytic and SERS performances of the as-prepared Au particles. The solution of p-ATP $(0.2 \mathrm{mM})$ presented very weak Raman signals at 1078 and $1588 \mathrm{~cm}^{-1}$, assigned to the stretching vibrations of $\mathrm{C}-\mathrm{S}$ and $\mathrm{C}-\mathrm{C}$ of $p$-ATP, respectively. ${ }^{41}$ In the presence of the sphere-like $\mathrm{Au}$ particles, prepared at $R_{\mathrm{Au} / \mathrm{APTES}}$ of $1: 2$, the signals were only enhanced slightly, corresponding to an 
enhanced factor $(E F)$ of $1 \times 10^{3}$. In the presence of the $\mathrm{Au}$ NFs prepared at $R_{\text {Au/APTES }}$ of $1: 8$, the signals were enhanced greatly, corresponding to an enhanced factor (EF) of $2 \times 10^{5}$, comparable to performance of most of the anisotropic $\mathrm{Au}$ particles in the literature (Table S1).,13-15,42-44 After the addition of $\mathrm{H}_{2} \mathrm{O}_{2}$, a new Raman band at $1430 \mathrm{~cm}^{-1}$, assigned to ring-stretching vibrations associated with the $\mathrm{N}=\mathrm{N}$ moiety, became observable (Figure 10a), indicating that the Au NFs are capable of catalyzing the dimerization of $p$-ATP into DMAB in the presence of $\mathrm{H}_{2} \mathrm{O}_{2}$, via conversion of the amino groups in $p$-ATP into $\mathrm{N}=\mathrm{N}$ bond induced by ${ }^{\bullet} \mathrm{OH}$ generated from the decomposition of $\mathrm{H}_{2} \mathrm{O}_{2} \cdot{ }^{41,45,46}$ In the time-dependent SERS spectra, which were normalized according to the signal of the $\mathrm{C}-\mathrm{S}$ bond at $1078 \mathrm{~cm}^{-1}$, the intensity of the signal at $1430 \mathrm{~cm}^{-1}$, assigned to the $\mathrm{N}=\mathrm{N}$ bond, increased gradually with the reaction time (Figure $10 \mathrm{~b}$ ), indicating the gradual conversion of $p$-ATP into DMAB with the proceeded reaction. The intensity ratio of the Raman signals at 1430 and 1078 $\mathrm{cm}^{-1}$, which may represent the amount of DMAB generated from the dimerization of $p$-ATP, ${ }^{47,48}$ increased with the reaction time and reached a platform within $5 \mathrm{~min}$. The results indicated that the dimerization of $p$-ATP can be monitored in situ using the Au NFs as a SERS substrate. Figure 10c further shows that the negative natural logarithm of the concentration of $p$-ATP, estimated from the intensity ratio at 1430 and 1078 $\mathrm{cm}^{-1}$, increases linearly with time at the early stage of the reaction, suggesting that the dimerization of $p$-ATP with the catalysis of the Au NFs is a first-order reaction ${ }^{46,49}$ with a rate constant of $(6.0 \pm 0.7) \times 10^{-2} \mathrm{~min}^{-1}$.

\section{CONCLUSIONS}

In summary, in this work, we demonstrated a facile approach for the synthesis of Au NFs using APTES as a shape-directing agent. With increasing the molar ratios of $\mathrm{HAuCl}_{4}$ to APTES, the morphology of the Au particles evolved from sphere-like to flower-like, accompanied by a red shift in $\lambda_{\max }$ of the LSPR peak of the Au particles from 520 to $685 \mathrm{~nm}$. In this approach, an increase in the concentration of APTES promotes the preferential growth of $\mathrm{Au}$ particles along the (111) facet, and thus the formation of $\mathrm{Au} \mathrm{NFs,} \mathrm{more} \mathrm{anisotropic} \mathrm{in} \mathrm{character.}$ The as-prepared $\mathrm{Au}$ NFs were qualified for simultaneously catalyzing the dimerization of $p$-ATP and monitoring the reaction via in situ SERS detection. It is expected that such an APTES-directed approach would highly benefit the development of Au NF-based chemical catalysis and SERS detection.

\section{EXPERIMENTAL SECTION}

Materials. Chlorauric acid tetrahydrate $\left(\mathrm{HAuCl}_{4} \cdot 4 \mathrm{H}_{2} \mathrm{O}\right.$, 99\%) was purchased from Sinopharm Chemical Reagent Co., Ltd. 3-Aminopropyltriethoxysilane (APTES, $\geq 98 \%$ ) was purchased from Sigma-Aldrich. Ascorbic acid (AA, $\geq 99 \%$ ) was purchased from Alfa Aesar and $p$-aminothiophenol ( $p$ ATP, 97\%) was purchased from Aladdin Industrial Incorporated. Hydrogen peroxide $\left(\mathrm{H}_{2} \mathrm{O}_{2}\right)$ and nitric acid $\left(\mathrm{HNO}_{3}\right)$ were purchased from Beijing Chemical Work and lithium hydroxide $(\mathrm{LiOH})$ was purchased from Tianjin Chemical Co. All reagents were analytical in grade and used without further purification. High-purity water (Pall Purelab Plus) with a resistivity of $18.2 \mathrm{M} \Omega \cdot \mathrm{cm}$ was used in all experiments. All glassware used was cleaned in a bath of freshly prepared aqua regia solution $\left(\mathrm{HCl} / \mathrm{HNO}_{3}, 3: 1\right)$ and a chromic acid lotion, and then rinsed thoroughly with high-purity water before use.
All of the experiments were carried out at room temperature $\left(25 \pm 2{ }^{\circ} \mathrm{C}\right)$ unless stated especially.

Characterization. UV-vis spectra were recorded on a Shimadzu UV-1800 UV-vis spectrophotometer. Temporal evolutions in UV-vis spectra were acquired using an Ocean Optics HR4000CG-UV-NIR high-resolution spectrophotometer. TEM images were taken on a JEOL JEM-2010 electron microscope with an acceleration voltage of $100 \mathrm{kV}$. HRTEM images, STEM images, and EDS measurements were taken on a JEOL JEM-2100F scanning transmission electron microscope with an acceleration voltage of $200 \mathrm{kV}$, equipped with a highangle annular dark-field (HAADF) detector. The dispersions of $\mathrm{Au}$ particles were dropped onto carbon-coated copper grids and then dried in air before the TEM and HRTEM observations. Mass spectrometric analysis was performed on a Thermo Fisher Orbitrap Fusion Tribrid mass spectrometer with negative-ion detection modes. Hydrodynamic diameters and $\zeta$-potentials were measured on a Brookhaven ZetaPALS apparatus. FTIR spectra were recorded using a Thermo Scientific Nicolet iS50 FTIR spectrophotometer in a scanning range from 500 to $2000 \mathrm{~cm}^{-1}$ with 64 scans at a resolution of 8 $\mathrm{cm}^{-1}$. The Au particles were collected by centrifugation and then dried in vacuum and mixed with $\mathrm{KBr}$ powders before FTIR measurements. XRD patterns were collected on a PANalytical Empyrean X-ray diffractometer equipped with a graphite monochromator ( $\mathrm{Cu} \mathrm{K} \alpha$ radiation, $\lambda=1.54 \AA$ ) at a scanning speed of $5^{\circ} \mathrm{min}^{-1}$. Raman and SERS spectra were acquired on a Horiba LabRAM HR Evolution spectrophotometer equipped with a $785 \mathrm{~nm}$ laser in a quartz cell with an optical path of $1 \mathrm{~cm}$. All of the Raman measurements were performed in the solution phase with an accumulation time of $60 \mathrm{~s}$ at a laser power of $50 \mathrm{~mW}$, which almost showed no photothermal effect on the dispersion of the Au NFs (Figure S6).

Preparation of Au Particles. APTES was added to highpurity water to form the $3 \mathrm{mM}$ aqueous solution and kept at room temperature for $5 \mathrm{~min}$. After that, $25 \mu \mathrm{L}$ of an aqueous solution of $\mathrm{HAuCl}_{4}(100 \mathrm{mM})$ and different volumes of the APTES aqueous solution $(3 \mathrm{mM})$ were mixed under magnetic stirring $(300 \mathrm{rpm})$ at room temperature with the final volume fixed at $10 \mathrm{~mL}$, followed by the addition of $150 \mu \mathrm{L}$ of an aqueous solution of AA $(100 \mathrm{mM})$. The concentrations of $\mathrm{HAuCl}_{4}$ and $\mathrm{AA}$ were set at 0.25 and $1.5 \mathrm{mM}$ and that of APTES varied from 0 to $0.5,1.0,1.5,2.0$, and $2.5 \mathrm{mM}$ in the final solutions, corresponding to the molar ratios of $\mathrm{HAuCl}_{4}$ and APTES $\left(R_{\mathrm{Au} / \mathrm{APTES}}\right)$ of $1: 0,1: 2,1: 4,1: 6,1: 8$, and 1:10, respectively. After $30 \mathrm{~min}$, the resulting Au particles were collected by centrifugation (3000 rpm, $10 \mathrm{~min}$ ) and then redispersed in pure water for further characterization.

Catalyzing and In Situ SERS Monitoring the Dimerization of $p$-ATP. Overall, $10 \mu \mathrm{L}$ of $0.2 \mathrm{mM} p$-ATP was added in $2 \mathrm{~mL}$ of a dispersion of $\mathrm{Au}$ particles $(0.02 \mathrm{nM})$ prepared at $R_{\text {Au/APTES }} 1: 2$ and 1:8, respectively, and then incubated for 10 $\mathrm{min}$ at room temperature. After transferring the abovementioned mixture into $1 \mathrm{~cm}$ quartz cuvettes, $0.50 \mathrm{mM}$ $\mathrm{H}_{2} \mathrm{O}_{2}$ was added into the mixture, followed by SERS measurements immediately.

The Raman enhancement factor (EF) was calculated using the following equation ${ }^{28}$

$$
\mathrm{EF}=\frac{I_{\text {SERS }}}{N_{\text {SERS }}} \times \frac{N_{\text {bulk }}}{I_{\text {bulk }}}
$$


where $I_{\text {SERS }}$ and $I_{\text {bulk }}$ are Raman intensities with and without $\mathrm{Au}$ particles and $N_{\text {SERS }}$ and $N_{\text {bulk }}$ are the number of probe molecules adsorbed on $\mathrm{Au}$ particles and in the bulk solution sample.

\section{ASSOCIATED CONTENT}

\section{SI Supporting Information}

The Supporting Information is available free of charge at https://pubs.acs.org/doi/10.1021/acsomega.1c03933.

Negative differentiation curve of the conductivity of the aqueous solution of APTES (Figure S1), normalized $\mathrm{UV}-$ vis spectrum and the TEM image of the $\mathrm{Au}$ particles prepared using fresh APTES (Figure S2), enlarged TEM images of the Au particles prepared at $R_{\text {Au/APTES }}$ of $1: 2$ and 1:8 (Figure S3), temporal evolution in hydrodynamic diameters and polydispersity of the $\mathrm{Au}$ NFs (Figure S4), variations in the hydrodynamic diameter and polydispersity of the Au NFs prepared from the five different batches (Figure S5), temperature change of dispersion of the $\mathrm{Au}$ NFs with the laser irradiation time (Figure S6), and brief summary of the SERS performance of anisotropic Au particles prepared in our work and those reported in the literature (Table S1) (PDF)

\section{AUTHOR INFORMATION}

\section{Corresponding Authors}

Zhenzhen Huang - State Key Laboratory of Inorganic Synthesis and Preparative Chemistry, College of Chemistry, Jilin University, Changchun 130012, China; (1) orcid.org/ 0000-0002-9300-5219; Email: huangzhen@jlu.edu.cn

Wensheng Yang - State Key Laboratory of Inorganic Synthesis and Preparative Chemistry, College of Chemistry, Jilin University, Changchun 130012, China; 이이.org/ 0000-0003-2674-6751; Email: wsyang@jlu.edu.cn

\section{Authors}

Jingwen Ba - State Key Laboratory of Inorganic Synthesis and Preparative Chemistry, College of Chemistry, Jilin University, Changchun 130012, China

Yandong Han - Institute of Molecular Plus, Tianjin University, Tianjin 300072, China

Xiaoyu Zhang - State Key Laboratory of Inorganic Synthesis and Preparative Chemistry, College of Chemistry, Jilin University, Changchun 130012, China

Lijuan Zhang - State Key Laboratory of Inorganic Synthesis and Preparative Chemistry, College of Chemistry, Jilin University, Changchun 130012, China

Shuhan Hui - State Key Laboratory of Inorganic Synthesis and Preparative Chemistry, College of Chemistry, Jilin University, Changchun 130012, China

Complete contact information is available at:

https://pubs.acs.org/10.1021/acsomega.1c03933

\section{Notes}

The authors declare no competing financial interest.

\section{ACKNOWLEDGMENTS}

This work was supported by the Natural Science Foundation of China (No. 21773089) and the Natural Science Foundation of Jilin Province of China (20190103116JH).

\section{REFERENCES}

(1) Reguera, J.; Langer, J.; Jiménez de Aberasturi, D.; Liz-Marzán, L. M. Anisotropic Metal Nanoparticles for Surface Enhanced Raman Scattering. Chem. Soc. Rev. 2017, 46, 3866-3885.

(2) Priecel, P.; Salami, H. A.; Padilla, R. H.; Zhong, Z.; LopezSanchez, J. A. Anisotropic Gold Nanoparticles: Preparation and Applications in Catalysis. Chin. J. Catal. 2016, 37, 1619-1650.

(3) Ye, S.; Benz, F.; Wheeler, M. C.; Oram, J.; Baumberg, J. J.; Cespedes, O.; Christenson, H. K.; Coletta, P. L.; Jeuken, L. J.; Markham, A. F.; Critchley, K.; Evans, S. D. One-Step Fabrication of Hollow-Channel Gold Nanoflowers with Excellent Catalytic Performance and Large Single-Particle SERS Activity. Nanoscale 2016, 8, 14932-14942.

(4) Lu, X.; Huang, Y.; Liu, B.; Zhang, L.; Song, L.; Zhang, J.; Zhang, A.; Chen, T. Light-Controlled Shrinkage of Large-Area Gold Nanoparticle Monolayer Film for Tunable SERS Activity. Chem. Mater. 2018, 30, 1989-1997.

(5) Xie, J.; Zhang, Q.; Lee, J. Y.; Wang, D. I. C. The Synthesis of SERS-Active Gold Nanoflower Tags for in Vivo Applications. ACS Nano 2008, 2, 2473-2480.

(6) Ma, H.; Liu, Z.; Wei, Y.; Jiang, L. Controlled Morphology Evolution of Branched $\mathrm{Au}$ Nanostructures and Their ShapeDependent Catalytic and Photo-Thermal Properties. Colloids Surf., A 2019, 582, No. 123889.

(7) Wang, L.; Liu, G.; Han, J.; Li, R.; Liu, J.; Chen, K.; Huang, M. One-Pot Synthesis of 3D Au Nanoparticle Clusters with Tunable Size and Their Application. Nanotechnology 2020, 31, No. 085601.

(8) Ahijado-Guzmán, R.; Sánchez-Arribas, N.; Martínez-Negro, M.; González-Rubio, G.; Santiago-Varela, M.; Pardo, M.; Piñeiro, A.; López-Montero, I.; Junquera, E.; Guerrero-Martínez, A. Intercellular Trafficking of Gold Nanostars in Uveal Melanoma Cells for Plasmonic Photothermal Therapy. Nanomaterials 2020, 10, No. 590.

(9) Djafari, J.; Fernández-Lodeiro, A.; García-Lojo, D.; FernándezLodeiro, J.; Rodríguez-González, B.; Pastoriza-Santos, I.; Pérez-Juste, J.; Capelo, J. L.; Lodeiro, C. Iron(II) as a Green Reducing Agent in Gold Nanoparticle Synthesis. ACS Sustainable Chem. Eng. 2019, 7, 8295-8302.

(10) Liu, K.; Han, L.; Zhuang, J.; Yang, D.-P. Protein-Directed Gold Nanoparticles with Excellent Catalytic Activity for 4-Nitrophenol Reduction. Mater. Sci. Eng., C 2017, 78, 429-434.

(11) Cheng, Q.; Song, L.; Lin, H.; Yang, Y.; Huang, Y.; Su, F.; Chen, T. Free-Standing 2D Janus Gold Nanoparticles Monolayer Film with Tunable Bifacial Morphologies via the Asymmetric Growth at Air Liquid Interface. Langmuir 2020, 36, 250-256.

(12) Shao, H.; Lin, H.; Guo, Z.; Lu, J.; Jia, Y.; Ye, M.; Su, F.; Niu, L.; Kang, W.; Wang, S.; Hu, Y.; Huang, Y. A Multiple Signal Amplification Sandwich-Type SERS Biosensor for Femtomolar Detection of Mirna. Biosens. Bioelectron. 2019, 143, No. 111616.

(13) Liebig, F.; Henning, R.; Sarhan, R. M.; Prietzel, C.; Schmitt, C. N. Z.; Bargheer, M.; Koetz, J. A Simple One-Step Procedure to Synthesise Gold Nanostars in Concentrated Aqueous Surfactant Solutions. RSC Adv. 2019, 9, 23633-23641.

(14) Song, C. Y.; Yang, B. Y.; Chen, W. Q.; Dou, Y. X.; Yang, Y. J.; Zhou, N.; Wang, L. H. Gold Nanoflowers with Tunable Sheet-Like Petals: Facile Synthesis, SERS Performances and Cell Imaging. J. Mater. Chem. B 2016, 4, 7112-7118.

(15) Zhang, Y.; Wang, B.; Yang, S.; Li, L.; Guo, L. Facile Synthesis of Spinous-Like $\mathrm{Au}$ Nanostructures for Unique Localized Surface Plasmon Resonance and Surface-Enhanced Raman Scattering. New J. Chem. 2015, 39, 2551-2556.

(16) Zhang, J. H.; Xi, C. X.; Feng, C.; Xia, H. B.; Wang, D. Y.; Tao, X. T. High Yield Seedless Synthesis of High-Quality Gold Nanocrystals with Various Shapes. Langmuir 2014, 30, 2480-2489.

(17) Zhong, L.; Zhai, X.; Zhu, X.; Yao, P.; Liu, M. Vesicle-Directed Generation of Gold Nanoflowers by Gemini Amphiphiles and the Spacer-Controlled Morphology and Optical Property. Langmuir 2010, $26,5876-5881$. 
(18) Dinda, E.; Rashid, M. H.; Mandal, T. K. Amino Acid-Based Redox Active Amphiphiles to In Situ Synthesize Gold Nanostructures: From Sphere to Multipod. Cryst. Growth Des. 2010, 10, 2421-2433.

(19) Pastoriza-Santos, I.; Liz-Marzán, L. M. N,N-Dimethylformamide as a Reaction Medium for Metal Nanoparticle Synthesis. Adv. Funct. Mater. 2009, 19, 679-688.

(20) Song, L.; Huang, Y.; Nie, Z.; Chen, T. Macroscopic TwoDimensional Monolayer Films of Gold Nanoparticles: Fabrication Strategies, Surface Engineering and Functional Applications. Nanoscale 2020, 12, 7433-7460.

(21) Song, C.; Zhou, N.; Yang, B.; Yang, Y.; Wang, L. Facile Synthesis of Hydrangea Flower-Like Hierarchical Gold Nanostructures with Tunable Surface Topographies for Single-Particle SurfaceEnhanced Raman Scattering. Nanoscale 2015, 7, 17004-17011.

(22) Vijayaraghavan, P.; Liu, C.-H.; Hwang, K. C. Synthesis of Multibranched Gold Nanoechinus Using a Gemini Cationic Surfactant and Its Application for Surface Enhanced Raman Scattering. ACS Appl. Mater. Interfaces 2016, 8, 23909-23919.

(23) Kariuki, V. M.; Hoffmeier, J. C.; Yazgan, I.; Sadik, O. A. Seedless Synthesis and SERS Characterization of Multi-Branched Gold Nanoflowers Using Water Soluble Polymers. Nanoscale 2017, 9, $8330-8340$.

(24) Huang, Y.; Dandapat, A.; Kim, D.-H. Covalently Capped SeedMediated Growth: A Unique Approach toward Hierarchical Growth of Gold Nanocrystals. Nanoscale 2014, 6, 6478-6481.

(25) Tamoto, R.; Lecomte, S.; Si, S.; Moldovan, S.; Ersen, O.; Delville, M.-H.; Oda, R. Gold Nanoparticle Deposition on Silica Nanohelices: A New Controllable 3D Substrate in Aqueous Suspension for Optical Sensing. J. Phys. Chem. C 2012, 116, 23143-23152.

(26) Sareen, S.; Mutreja, V.; Singh, S.; Pal, B. Highly Dispersed Au, $\mathrm{Ag}$ and $\mathrm{Cu}$ Nanoparticles in Mesoporous SBA-15 for Highly Selective Catalytic Reduction of Nitroaromatics. RSC Adv. 2015, 5, 184-190.

(27) Zhang, M.; Liu, Y.; Jia, P.; Feng, Y.; Fu, S.; Yang, J.; Xiong, L.; $\mathrm{Su}, \mathrm{F}$; $\mathrm{Wu}, \mathrm{Y}$.; Huang, Y. Ag Nanoparticle-Decorated Mesoporous Silica as a Dual-Mode Raman Sensing Platform for Detection of Volatile Organic Compounds. ACS Appl. Nano Mater. 2021, 4, 10191028.

(28) Le Ru, E. C.; Blackie, E.; Meyer, M.; Etchegoin, P. G. Surface Enhanced Raman Scattering Enhancement Factors: A Comprehensive Study. J. Phys. Chem. C 2007, 111, 13794-13803.

(29) Howarter, J. A.; Youngblood, J. P. Optimization of Silica Silanization by 3-Aminopropyltriethoxysilane. Langmuir 2006, 22, 11142-11147.

(30) Peña-Alonso, R.; Rubio, F.; Rubio, J.; Oteo, J. L. Study of the Hydrolysis and Condensation of Gamma-Aminopropyltriethoxysilane by FT-IR Spectroscopy. J. Mater. Sci. 2007, 42, 595-603.

(31) Chen, S.-L.; Dong, P.; Yang, G.-H.; Yang, J.-J. Kinetics of Formation of Monodisperse Colloidal Silica Particles through the Hydrolysis and Condensation of Tetraethylorthosilicate. Ind. Eng. Chem. Res. 1996, 35, 4487-4493.

(32) Han, Y.; Lu, Z.; Teng, Z.; Liang, J.; Guo, Z.; Wang, D.; Han, M.-Y.; Yang, W. Unraveling the Growth Mechanism of Silica Particles in the Stöber Method: In Situ Seeded Growth Model. Langmuir 2017, 33, 5879-5890.

(33) Dong, H.; Han, Y.; Teng, S.; Hu, Y.; Guo, Z.; Wang, D.; Yang, W. Tetrabutylammonium Bromide Assisted Preparation of Monodispersed Submicrometer Silica Particles. Colloids Surf., A 2021, 614, No. 126171.

(34) Guan, Y.; Xue, Z.; Liang, J.; Huang, Z.; Yang, W. One-Pot Synthesis of Size-Tunable Hollow Gold Nanoshells via APTES-inWater Suspension. Colloids Surf., A 2016, 502, 6-12.

(35) Zhao, L.; Ji, X.; Sun, X.; Li, J.; Yang, W.; Peng, X. Formation and Stability of Gold Nanoflowers by the Seeding Approach: The Effect of Intraparticle Ripening. J. Phys. Chem. C 2009, 113, 1664516651

(36) Abbasi, Z.; Saeed, W.; Shah, S. M.; Shahzad, S. A.; Bilal, M.; Khan, A. F.; Shaikh, A. J. Binding Efficiency of Functional Groups
Towards Noble Metal Surfaces Using Graphene Oxide - Metal Nanoparticle Hybrids. Colloids Surf., A 2021, 611, No. 125858.

(37) Majoul, N.; Aouida, S.; Bessaïs, B. Progress of Porous Silicon APTES-Functionalization by FTIR Investigations. Appl. Surf. Sci. 2015, 331, 388-391.

(38) Sun, X.; Wei, W. Electrostatic-Assembly-Driven Formation of Micrometer-Scale Supramolecular Sheets of (3-Aminopropyl)Triethoxysilane (APTES)-HAuCl 4 and Their Subsequent Transformation into Stable APTES Bilayer-Capped Cold Nanoparticles through a Thermal Process. Langmuir 2010, 26, 6133-6135.

(39) Wu, Z.; Xiang, H.; Kim, T.; Chun, M.-S.; Lee, K. Surface Properties of Submicrometer Silica Spheres Modified with Aminopropyltriethoxysilane and Phenyltriethoxysilane. J. Colloid Interface Sci. 2006, 304, 119-124.

(40) Grirrane, A.; Corma, A.; García, H. Gold-Catalyzed Synthesis of Aromatic Azo Compounds from Anilines and Nitroaromatics. Science 2008, 322, 1661-1664.

(41) Huang, Y.-F.; Wu, D.-Y.; Zhu, H.-P.; Zhao, L.-B.; Liu, G.-K.; Ren, B.; Tian, Z.-Q. Surface-Enhanced Raman Spectroscopic Study of p-Aminothiophenol. Phys. Chem. Chem. Phys. 2012, 14, 8485-8497.

(42) Chen, L.-X.; Lv, J.-J.; Wang, A.-J.; Huang, H.; Feng, J.-J. OneStep Wet-Chemical Synthesis of Gold Nanoflower Chains as Highly Active Surface-Enhanced Raman Scattering Substrates. Sens. Actuators, B 2016, 222, 937-944.

(43) Jeong, S.; Kim, M.-W.; Jo, Y.-R.; Kim, N.-Y.; Kang, D.; Lee, S. Y.; Yim, S.-Y.; Kim, B.-J.; Kim, J. H. Hollow Porous Gold Nanoshells with Controlled Nanojunctions for Highly Tunable Plasmon Resonances and Intense Field Enhancements for Surface-Enhanced Raman Scattering. ACS Appl. Mater. Interfaces 2019, 11, 4445844465 .

(44) Lin, G.; Xian, L.; Zhou, X.; Wang, S.; Shah, Z. H.; Edwards, S. A.; Gao, Y. Design and One-Pot Synthesis of Capsid-Like Gold Colloids with Tunable Surface Roughness and Their Enhanced Sensing and Catalytic Performances. ACS Appl. Mater. Interfaces 2020, $12,50152-50160$.

(45) Yang, X.-J.; Tian, P.-F.; Wang, H.-L.; Xu, J.; Han, Y.-F. Catalytic Decomposition of $\mathrm{H}_{2} \mathrm{O}_{2}$ over a Au/Carbon Catalyst: A Dual Intermediate Model for the Generation of Hydroxyl Radicals. J. Catal. 2016, 336, 126-132.

(46) Cui, K.; Fan, C.; Chen, G.; Qiu, Y.; Li, M.; Lin, M.; Wan, J.-B.; Cai, C.; Xiao, Z. Para-Aminothiophenol Radical Reaction-Functionalized Gold Nanoprobe for One-to-All Detection of Five Reactive Oxygen Species in Vivo. Anal. Chem. 2018, 90, 12137-12144.

(47) Pal, S.; Dutta, A.; Paul, M.; Chattopadhyay, A. PlasmonEnhanced Chemical Reaction at the Hot Spots of End-to-End Assembled Gold Nanorods. J. Phys. Chem. C 2020, 124, 3204-3210.

(48) da Silva, A. G. M.; Rodrigues, T. S.; Wang, J.; Yamada, L. K.; Alves, T. V.; Ornellas, F. R.; Ando, R. A.; Camargo, P. H. C. The Fault in Their Shapes: Investigating the Surface-Plasmon-ResonanceMediated Catalytic Activities of Silver Quasi-Spheres, Cubes, Triangular Prisms, and Wires. Langmuir 2015, 31, 10272-10278.

(49) Panigrahi, S.; Basu, S.; Praharaj, S.; Pande, S.; Jana, S.; Pal, A.; Ghosh, S. K.; Pal, T. Synthesis and Size-Selective Catalysis by Supported Gold Nanoparticles: Study on Heterogeneous and Homogeneous Catalytic Process. J. Phys. Chem. C 2007, 111, $4596-4605$ 